\title{
Effects of $\mathrm{LI11}$ Acupuncture on $\mathrm{CO}_{2}$ reactivity in the anterior and middle cerebral arteries during hyperventilation-induced hypocapnia in normal subjects: A before and after study.
}

\author{
Gyeong-muk Kim, Woo-Sang Jung\#, Seungwon Kwon*, Chul Jin, Seung-Yeon Cho, Seong-Uk Park, \\ Sang-Kwan Moon, Jung-Mi Park, Chang-Nam Ko, Ki-Ho Cho \\ Department of Cardiology and Neurology, College of Korean Medicine, Kyung Hee University
}

\begin{abstract}
Objectives: The LI11 (Quchi) acupuncture point has always been included in the Seven acupoints for stroke; however, additional LI11 acupuncture research is needed. In this study, the effect of LI11 acupuncture on cerebral blood flow of the anterior cerebral arteries (ACA) and middle cerebral arteries (MCA) was investigated.

Method: This study included 10 healthy young male subjects. Cerebral blood flow velocity and cerebrovascular reactivity were measured using transcranial Doppler sonography. Changes in hyperventilation-induced carbon dioxide $\left(\mathrm{CO}_{2}\right)$ reactivity and modified ACA and MCA blood flow velocity at $40 \mathrm{mmHg}(\mathrm{CV} 40)$, blood pressure, and heart rate were observed before and after LI11 acupuncture treatment.

Results: A statistically significant increase in contralateral anterior cerebral artery $\mathrm{CO}_{2}$ reactivity $(\mathrm{p}=0.036)$ and decrease in contralateral middle cerebral artery CV40 $(\mathrm{p}=0.047)$ were observed. No significant difference in mean blood pressure was shown. A statistically significant increase in heart rate occurred after LI11 acupuncture; however, it was not clinically significant as there were negligible changes in the heart rhythm.

Conclusions: LI11 acupuncture treatment could improve cerebral blood flow velocity. These results might be explained by regulating endothelium-dependent vessel dilation in the anterior cerebral artery region.

Trial registration: This trial has been registered with Clinical Research Information Service, a service of the Korea Centers for Disease Control and Prevention: KCT0004494 (retrospectively registered). https://cris.nih.go.kr/cris/search/ search_result_st01.jsp?seq=15359
\end{abstract}

$\overline{K e y}$ Words : LI11; transcranial Doppler sonography; anterior cerebral artery; middle cerebral artery; cerebrovascular reactivity; cerebral blood flow velocity

\section{Introduction}

Acupuncture has been used to treat various diseases for thousands of years ${ }^{1)}$. Nowadays, acupuncture treatment is applied to mediate the effects of a stroke, including hemiparesis, ${ }^{2)}$ rigidity, ${ }^{3)}$ and dysphagia. ${ }^{4)}$ These therapeutic effects may be due to an increase in cerebral blood flow, as observed using positron emission tomography (PET), single-photon emission

- Received : 5 October 2021

- Accepted : 2 November 2021

- Correspondence to : Seungwon Kwon

Department of Cardiology and Neurology, College of Korean Medicine, Kyung Hee University

23 Kyungheedae-ro, Dongdaemun-gu, Seoul 02447, Korea

Tel : +82-2-958-9128, Fax : +82-2-958-9132, E-mail : kkokkottung@hanmail.net

\# These authors are equally contributed to this manuscript as first authors 
computed tomography (SPECT), and transcranial doppler imaging techniques.

In Traditional East Asian Medicine, the "Seven Acupoints for Stroke" are acupuncture points that are used to treat stroke symptoms. Although the composition of acupuncture points has changed over time, LI11 has always been included. ${ }^{5}$ Current studies indicate that LI11 acupuncture can improve symptoms and sequelae of stroke as a means of rehabilitation. ${ }^{6-7)}$ Several clinical trials have been conducted to evaluate the effect of acupuncture treatment on motor recovery after stroke. In those clinical trials, LI11 is one of the most used acupuncture points with LI4, LI10, and GB34. ${ }^{8)}$ The clinical mechanism of LI11 stimulation, however, is not clearly understood.

Some experiments have evaluated the effect of LI11 acupuncture on cerebral blood flow. Moon $(2004)^{9)}$ and Ryu (2006) ${ }^{10)}$ used electroacupuncture at LI4-LI11 and used SPECT to verify changes in cerebral blood flow. They found cerebral blood flow improvement in the frontal and temporal lobes, which included anterior cerebral artery vessel territory. Cho (2010) ${ }^{11)}$ conducted acupuncture at LI11-ST36 and used fMRI to examine the brain activating site. They found that acupuncture stimulation including LI11 activated the contralateral parietal lobe, midbrain, and nodule. However, the previous studies did not evaluate the effects of LI11 alone. Therefore, it is difficult to conclude that the above results are entirely due to LI11 acupuncture treatment.In this study, the effects of LI11 acupuncture on cerebral blood flow and carbon dioxide $\left(\mathrm{CO}_{2}\right)$ reactivity were studied using transcranial doppler. Transcranial doppler was selected as it is an inexpensive, low-risk, and easy-to-administer bedside procedure. ${ }^{12)}$ It can also noninvasively measure cerebral blood flow velocity in the main intracranial vessels with high accuracy. ${ }^{13)}$ In addition, a previous study suggested that $\mathrm{CO}_{2}$ reactivity measured by transcranial doppler has a significant association with internal borderzone infarction of patients with internal carotid artery occlusion. ${ }^{14)}$ In several previous studies, transcranial doppler was used to find alterations in cerebral blood flow after acupuncture stimulation to specific acupuncture points, namely GV20, ${ }^{15)}$ GB20, ${ }^{16)}$ ST36, ${ }^{17)}$ and GB34 ${ }^{18)}$ which have been frequently used in acupuncture treatment for patients with cerebrovascular disease. The cerebral arteries, which showed significant changes according to the acupuncture points used in each study, differed, ${ }^{15-18)}$ which is presumed to be the result of specificity of acupuncture points. Research on the specificity of the acupuncture point has been conducted in many ways, which might be valuable for enhancing practical acupuncture treatment. $^{19)}$

Therefore, to investigate the possibility of LI11 in acupuncture treatment for patients with cerebrovascular disease and specificity of LI11 on cerebral artery, this study was conducted to assess the effects of LI11 acupuncture on the cerebral artery using transcranial doppler.

\section{Materials and Methods}

\section{Subjects and Acupuncture treatment at LI11}

This is a single center, before-and-after design, and pilot study. All study procedure was conducted 
at Kyung Hee University Korean Medicine Hospital, Seoul, Korea. Ten healthy male subjects were enrolled in this study. Prior to the study, all subjects were informed of the procedures and signed a written agreement. Subjects who had a history of cerebrovascular, cardiovascular, or endocrine disease, diabetes mellitus, hypertension, or psychiatric problems and had any disease at the time of study were excluded. After enrollment, all subjects were forbidden to drink caffeine and alcohol or take any medicine 24 hours before the study.

All acupuncture treatment procedures were performed by a well-trained Korean medicine doctor with more than three years' experience. The treated acupuncture point, LI11, is located on the dorsal radial side of the forearm, on the line connecting LI5 and LI11, $2 \mathrm{~cm}$ below the transverse cubital crease while the elbow is flexed. This landmark of LI11 location follows the WHO standard for acupuncture point locations (Figure 1). For LI11 acupuncture on the

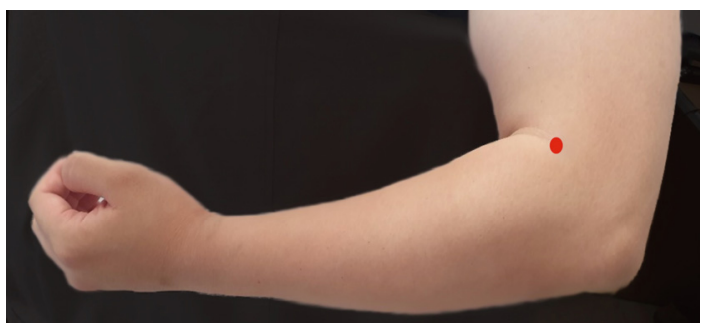

Fig. 1. Location of LI11

right side, a stainless-steel acupuncture needle (diameter $0.25 \mathrm{~mm}$, length $40 \mathrm{~mm}$; DongBang Acupuncture, Seoul, Korea) was used. Manual stimulation was used until subjects felt the De-Qi sensation. The needle was inserted into the skin to a depth of approximately $20 \mathrm{~mm}$, and the needle was removed after 20 minutes. All procedures followed the Revised Standards for Reporting Interventions in Clinical Trials of Acupuncture (STRICTA, ${ }^{20)}$ Table 1).

This study was approved by the Institutional Review Board at the Kyung Hee University Korean Medicine Hospital (KOMCIRB-2018 $-08-004-001)$.

Table 1. Acupuncture rationale and needling details according to the STRICTA guideline

\begin{tabular}{|c|c|}
\hline Items & Contents \\
\hline 1. Acupuncture rationale & $\begin{array}{l}\text { 1a) According to meridian theory of Traditional Korean medicine } \\
\text { 1b) Based on historical context - Seven acupoints for stroke }\end{array}$ \\
\hline 2. Details of needling & $\begin{array}{l}\text { 2a) } 1 \text { needle } \\
\text { 2b) Quchi (LI11) } \\
\text { 2c) } 20 \mathrm{~mm} \\
\text { 2d) De-qi sensation } \\
\text { 2e) Manual stimulation } \\
\text { 2f) } 20 \text { minutes } \\
\text { 2g) Stainless steel acupuncture (diameter } 0.25 \mathrm{~mm} \text {, length } 40 \mathrm{~mm} \text {, DongBang Acupuncture, } \\
\text { Seoul, Korea) }\end{array}$ \\
\hline 3. Treatment regimen & $\begin{array}{l}\text { 3a) } 2 \text { sessions (once per week) } \\
\text { 3b) Duration, } 1 \text { week }\end{array}$ \\
\hline 4. Other components of Treatment & 4a) None \\
\hline 5. Practitioner background & 5a) All acupuncture treatment was performed by one experienced Korean medical doctor \\
\hline
\end{tabular}




\section{Measurements and Statistical analysis}

The process and sample size $(n=10)$ of this study was based on previous studies that aimed to identify relationships between $\mathrm{CO}_{2}$ reactivity and other acupuncture points. ${ }^{15-18)}$ For each process, cerebral blood flow velocity and $\mathrm{CO}_{2}$ reactivity of both anterior cerebral arteries and middle cerebral arteries were measured using a transcranial doppler machine (Multi-Dop X4 system, Compumedics DWL, Singen, Germany).

Each subject was tested twice approximately one week apart. During the first visit, all subjects wore a measurement device (Figure 2) while in a comfortable supine position. Middle cerebral artery cerebral blood flow velocities were collected during both temporal windows using a $2 \mathrm{MHz}$ pulsed-Doppler probe. During the second visit, all subjects also wore a measurement device in a supine position and anterior cerebral artery cerebral blood flow velocities were collected using the same procedure. This measurement device consists of a removable bilateral probe holder (LAM-Rack; Compumedics DWL, Singen, Germany), and a probe settled at both temporal windows to maintain consistent data collection.

Every cerebral blood flow measurement began after 5 minutes of quiet sitting. Cerebral blood flow velocities and $\mathrm{CO}_{2}$ reactivity of all anterior cerebral arteries and middle cerebral arteries were measured at rest, and then during 1 minute of hypocapnia induced by hyperventilation. Hyperventilation was induced by over-breathing. The strongest wave pattern was captured at depths ranging from 65 to $80 \mathrm{~mm}$ for anterior cerebral arteries and 45 to $60 \mathrm{~mm}$ for middle cerebral arteries. Sample and gain values were

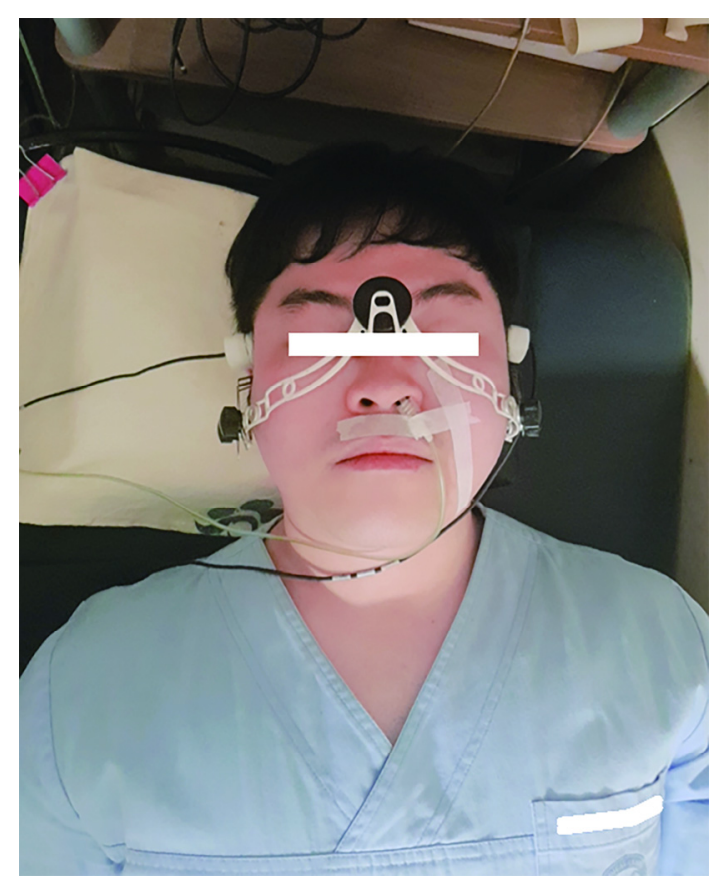

Fig. 2. Wearing a measuring tool, probe holder

corrected and saved when cerebral blood flow wave patterns remained constant. Mean blood flow velocity was calculated continuously as the time-averaged maximum velocity over the cardiac cycle as computed from the envelope of maximum frequencies. Mean anterior cerebral artery and middle cerebral artery blood flow velocities were obtained at rest under stable normocapnic conditions, and near the end of the hyperventilation period under hypocapnic conditions. All transcranial doppler spectra were recorded for later review.

Cerebral blood flow velocity is dependent on arterial $\mathrm{CO}_{2}$ tension, and corrected blood flow velocity was calculated at $40 \mathrm{mmHg}$ of $\mathrm{CO}_{2}$ tension (CV40, cm/s) using the following formula: 


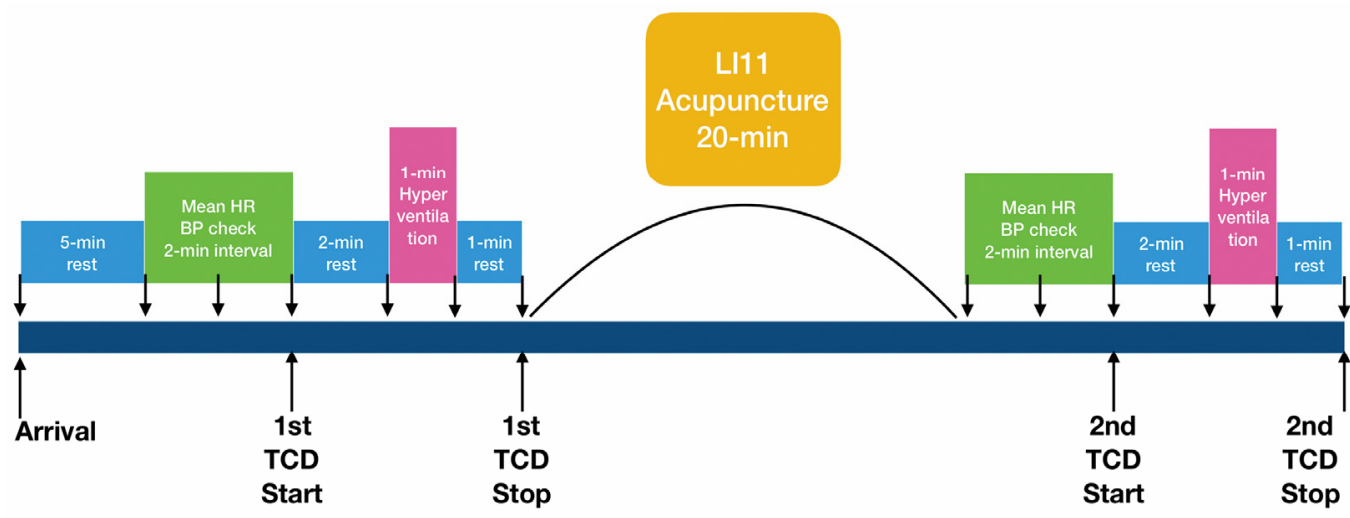

Fig. 3. Timeline of study procedure

CV40 (Corrected Velocity at $\mathrm{P}_{\mathrm{ETCO} 2} 40 \mathrm{mmHg}$ )

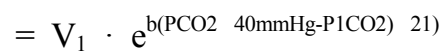

where $\mathrm{b}$ is $\mathrm{CO}_{2}$ reactivity, $\mathrm{V}_{1}$ is velocity at $\mathrm{P}_{1 C O}$, and $\mathrm{P}_{\mathrm{ETCO} 2}$, is end-tidal $\mathrm{CO}_{2}$ partial pressure.

$$
\mathrm{CO}_{2} \text { reactivity }=
$$

$\mathrm{CO}_{2}$ reactivity refers to the percent change in mean blood flow velocity per millimetre of mercury change in $\mathrm{P}_{\mathrm{ETCO} 2}$, as calculated by the above formula ${ }^{22)}$ where $V_{\text {rest }}$ is blood flow velocity at rest obtained during the most stable period under stable normocapnic conditions. $\mathrm{V}_{\text {hypocapnia }}$ is blood flow velocity in the latter half of the 1 minute hyperventilation period and $\Delta$ $\mathrm{P}_{\mathrm{ETCO} 2}$ is change in $\mathrm{P}_{\mathrm{ETCO} 2}$ from baseline to maximal hyperventilation. $\mathrm{CO}_{2}$ reactivity is expressed as \%/minute.

Using various modules of the Cardiocap $\mathrm{S} / 5$ collector (Datex-Ohmeda, Helsinki, Finland), variables that can affect cerebral blood flow such as blood pressure, heart rate, $\mathrm{P}_{\mathrm{ETCO} 2}$, and transcutaneous (tc) $\mathrm{PO}_{2}$ were measured. Blood pressure was measured three times at 2-minute intervals after the subject's condition stabilized. Blood pressure values were all used to calculate mean blood pressure. Heart rate and $\mathrm{tcPO}_{2}$ were continuously recorded through an oximetry device located on the subject's finger. Further, $\mathrm{P}_{\mathrm{ETCO} 2}$ was continuously obtained by a Cardiocap $\mathrm{S} / 5$ collector-connected nasal prong located in the subject's nostril, and each subject was instructed to breathe only through the nose during the procedure. A snapshot function in the Cardiocap $\mathrm{S} / 5$ collector program was used to obtain mean heart rate, $\mathrm{P}_{\mathrm{ETCO} 2}$, and mean $\mathrm{tcPO}_{2}$ at specific time points during the procedure. These variables were monitored and recorded on a computer connected to the Cardiocap $\mathrm{S} / 5$ collector program. Before and after the 20-minute LI11 acupuncture treatment, all measurements were performed using the above tools. A timeline of the study procedure can be found in Figure 3.

Statistical analysis was performed using the Statistical Package for the Social Sciences version 12.0 for Windows (SPSS, Chicago, Illinois, United States). Statistical comparisons between 
values before and after LI11 acupuncture treatment were made using the Wilcoxon signed-rank test, and p-values under 0.05 were considered statistically significant. Data are summarized as median (range).

\section{Results}

From October 2018 to September 2019, 10 healthy male subjects (26.431.23 years) were recruited and investigated (Table 2).

Table 2. Baseline characteristics

\begin{tabular}{ll}
\hline Items & Contents \\
\hline Sex (Male $:$ Female) & $10: 0$ \\
Age (years, mean \pm SD) & 26.431 .23 \\
\hline SD: Standard Deviation &
\end{tabular}

\section{Changes in $\mathrm{CO}_{2}$ reactivity after $\mathrm{LI} 11$ acupuncture}

When compared to baseline values, there was a statistically significant increase in $\mathrm{CO}_{2}$ reactivity of the contralateral anterior cerebral artery after LI11 acupuncture treatment $(P=0.036)$. However, there were no significant changes in ipsilateral anterior cerebral artery or middle cerebral artery, or contralateral middle cerebral artery $\mathrm{CO}_{2}$ reactivity pre and post-treatment. There were also no significant changes in anterior cerebral artery or ipsilateral middle cerebral artery CV40 pre and post-treatment (Table 3).

\section{Changes in CV40, mean blood pressure, and heart rate}

There was a significant decrease in contralateral middle cerebral artery CV40 $(p=0.047)$. There were no significant changes in CV40 in both anterior cerebral arteries and ipsilateral middle cerebral artery as a result of treatment (Table 3). Mean heart rate was slightly increased and there was no significant difference in blood pressure (Table 3).

During the entire trial, no specific adverse events from acupuncture were identified.

\section{Discussion}

In this study, LI11 acupuncture treatment improved $\mathrm{CO}_{2}$ reactivity of the contralateral anterior cerebral artery and had no significant effect on $\mathrm{CO}_{2}$ reactivity in both middle cerebral arteries or the ipsilateral anterior cerebral artery. Only the CV40 of the contralateral middle cerebral artery decreased while no significant change in the both anterior cerebral arteries or ipsilateral middle cerebral artery was observed.

Cerebral vasomotor reactivity was used to evaluate the compensatory potential of the vessels regulating blood flow to the brain, and is represented as the percentage change in response to an arteriolar-dilating stimulus such as $\mathrm{CO}_{2}$ or acetazolamide. ${ }^{23-24)}$ In this study, the hyperventilation -induced hypocapnia method was applied to measure $\mathrm{CO}_{2}$ reactivity. ${ }^{22)}$ Cerebral blood flow, through changing vessel diameter, can be divided into endothelium-dependent and non-dependent. Based on previous studies, cerebral blood flow and cerebrovascular reactivity (CVR) are regarded as being endothelium dependent. ${ }^{25-26)}$ Endothelium -dependent cerebral blood flow regulation is induced by NO or Endothelin-1, which alter blood vessel diameter. Endothelium dependence is considered to be affected by acupuncture 
stimulation.

In the present study, we performed acupuncture treatment only on the LI11 of right side. Assuming that two LI11 points of the left and right side will have the same effect, the effect of acupuncture treatment on the ipsilateral and contralateral side was evaluated. Our results show increasing $\mathrm{CO}_{2}$ reactivity of contralateral anterior cerebral artery and no change in both middle cerebral arteries or ipsilateral anterior cerebral artery reactivity. CV40 of the contralateral middle cerebral artery significantly decreased, but CV40 of both anterior cerebral arteries and ipsilateral middle cerebral artery did not change. A previous study used SPECT to show that LI4-LI11 electroacupuncture improved cerebral blood flow in the frontal and anterior temporal lobes. ${ }^{9-10)}$ These results are in accordance with the results of this study. The large intestine meridian to which LI11 belongs crosses from upper lip to the opposite side at the philtrum, and ends at the lateral side of the nose. ${ }^{5)}$ This pathway is also consistent with the results of the present study, which showed an increase in $\mathrm{CO}_{2}$ reactivity of the contralateral anterior cerebral artery. Since reduction of $\mathrm{CO}_{2}$ reactivity measured by transcranial doppler is known to be significantly related to cerebral infarction of the corresponding vascular territory, ${ }^{14)}$ this result suggests that LI11 might be useful in the treatment of cerebral infarction in the anterior cerebral artery territory. In other studies, ${ }^{27-30)}$ acupuncture treatments are known to induce intravenous cells recovery, increased blood flow through the expansion of the brain's blood vessels, and active nNOS. An experimental study using transcranial doppler showed differences in cerebral blood flow area that affects blood transfusion. A significant increase in CVR and CV40 levels in both middle cerebral arteries and anterior cerebral arteries has

Table 3. Changes in Parameters before and after LI11 acupuncture

\begin{tabular}{|c|c|c|c|c|}
\hline Parameters & Vessels & Before & After & p-value \\
\hline \multirow{4}{*}{$\begin{array}{l}\mathrm{CO} 2 \text { reactivity } \\
(\% / \text { minute })\end{array}$} & Ipsilateral ACA & $0.86(0.56-1.87 / 0.95 \pm 0.39)$ & $0.86(0.74-2.21 / 1.04 \pm 0.44)$ & 0.126 \\
\hline & Contralateral ACA & $1.05(0.57-2.10 / 1.11 \pm 0.41)$ & $1.10(0.85-2.80 / 1.26 \pm 0.57)$ & 0.036 \\
\hline & Ipsilateral MCA & $2.60(1.65-3.33 / 2.59 \pm 0.44)$ & $2.47(2.19-3.65 / 2.60 \pm 0.47)$ & 0.646 \\
\hline & Contralateral MCA & $2.20(1.67-3.29 / 2.6 \pm 0.47)$ & $2.12(1.77-3.47 / 2.46 \pm 0.61)$ & 0.575 \\
\hline \multirow[t]{4}{*}{$\mathrm{CV} 40(\mathrm{~cm} / \mathrm{s})$} & Ipsilateral ACA & $42.42(34.03-68.17 / 46.33 \pm 12.33)$ & $44.65(34.32-65.40 / 46.59 \pm 10.80)$ & 0.953 \\
\hline & Contralateral ACA & $49.76(31.55-71.46 / 50.07 \pm 14.02)$ & $49.02(34.45-73.76 / 50.59 \pm 14.08)$ & 0.575 \\
\hline & Ipsilateral MCA & $70.16(59.37-83.91 / 71.73 \pm 8.19)$ & $67.41(51.50-79.02 / 66.93 \pm 8.57)$ & 0.139 \\
\hline & Contralateral MCA & $67.41(53.30-94.50 / 67.09 \pm 13.30)$ & $58.86(50.25-78.95 / 62.49 \pm 11.58)$ & 0.047 \\
\hline $\begin{array}{l}\text { Mean blood pressure } \\
(\mathrm{mmHg})\end{array}$ & & $90.67(77.00-116.00 / 90.03 \pm 10.14)$ & $88.00(74.67-114.67 / 89.24 \pm 9.86)$ & 0.135 \\
\hline $\begin{array}{l}\text { Heart rate } \\
(\mathrm{bpm})\end{array}$ & & $76.15(56.00-99.00 / 75.23 \pm 12.03)$ & $77.40(57.00-99.00 / 76.10 \pm 11.81)$ & 0.001 \\
\hline
\end{tabular}


been observed during GV20 acupuncture. ${ }^{15)}$ In a study of GB20 acupuncture in 2014, $\left.{ }^{16}\right)$ it was reported that cerebral blood flow of basilar artery, posterior cerebral artery, and vertebral artery increased. Because GB20 is located in the back of the cervix, it can affect posterior circulation. In a study of acupuncture at ST36, ${ }^{17)}$ CVR of middle cerebral artery and basilar artery were significantly increased. In the case of GB34, ${ }^{18)}$ CVR of ipsilateral middle cerebral artery was significant increased. In a study using conventional procedures, various changes in blood flow have been observed in different regions by stimulating different acupuncture points. These changes correlated with different acupuncture points and different acupuncture specificities indicating different cerebral activities. In a study using similar procedures, various changes in cerebral blood flow were observed according to the specificity of acupuncture points.

There were a few limitations of the study. Based on the characteristics of the transcranial doppler test, training and repeated conditions may have influenced the experimental results. It is also a limitation that demographic characteristics such as history of smoking, caffeine usage, alcohol intake, level of physical activity, and recent experience of acupuncture have not been investigated. Since the order of measurement of middle cerebral artery and anterior cerebral artery has not been randomized, it might not be possible to exclude the carry-over effect of acupuncture. In the future study, it should be conducted with more subjects under limited conditions. This study has shown a significant decrease in contralateral middle cerebral artery CV40; However, unlike the
$\mathrm{CO}_{2}$ reactivity of the contralateral anterior cerebral artery, which showed a significant increase, a significant decrease in CV40 of the middle cerebral artery is not supported by the results of previous studies. ${ }^{9-10)}$ The results of previous studies using SPECT only showed improved cerebral blood flow in the frontal and anterior temporal lobes, but the blood flow velocity did not decrease in this area. Therefore, we concluded that a statistical bias induced by a small number of subjects may exist. As such, a larger study is needed to verify these results. Although the p-value of the change in heart rate was under 0.05 , it could be considered as insignificant. In most of the cases, the changes were within only one beat per minute (bpm). Therefore, it can be suggested that acupuncture on LI11 did not affect heart rhythm. Extended studies for different ages and genders should be conducted to support the conclusions of this study. Finally, future studies should be conducted on patients with cerebrovascular disease with impaired $\mathrm{CO}_{2}$ reactivity. Since this study has a process of inducing hyperventilation, it could not be conducted for patients with cerebrovascular disease who have a risk such as recurrence of cerebrovascular disease. Therefore, future studies need to use evaluation tools such as SPECT for patients with cerebrovascular disease.

\section{Conclusions}

Overall, this study has shown that LI11 acupuncture treatment increased $\mathrm{CO}_{2}$ reactivity, specifically in the contralateral anterior cerebral artery, and decreased corrected velocity at $\mathrm{P}_{\mathrm{ETCO} 2}$ $40 \mathrm{mmHg}$ in the contralateral middle cerebral 
artery during hypocapnia in healthy subjects. These results indicate that LI11 acupuncture treatment might improve cerebral blood flow velocity by regulating endothelium-dependent vessel dilation in only the anterior cerebral artery.

\section{Acknowledgements}

This paper is based on Gyeong-muk Kim's theses for Master's Degree. Gyeong-muk Kim and Woo-Sang Jung are equally contributed to this manuscript as first authors.

\section{References}

1. Fan, A. Y., Miller, D. W., Bolash, B., Bauer, M., McDonald, J., Faggert, S., He, H., Li, Y. M., Matecki, A., Camardella, L., Koppelman, M. H., Stone, J., Meade, L., \& Pang, J. (2017). Acupuncture's Role in Solving the Opioid Epidemic: Evidence, Cost-Effectiveness, and Care Availability for Acupuncture as a Primary, Non-Pharmacologic Method for Pain Relief and Management-White Paper 2017. Journal of integrative medicine, 15(6), 411-425. https://doi.org/10.1016/S2095-4964 (17)60378-9

2. Chen, L., Fang, J., Ma, R., Gu, X., Chen, L., Li, J., \& Xu, S. (2016). Additional effects of acupuncture on early comprehensive rehabilitation in patients with mild to moderate acute ischemic stroke: a multicenter randomized controlled trial. BMC complementary and alternative medicine, 16, 226. https://doi.org/10. 1186/s12906-016-1193-y

3. Cai, Y., Zhang, C. S., Liu, S., Wen, Z.,
Zhang, A. L., Guo, X., Lu, C., \& Xue, C. C. (2017). Electroacupuncture for Poststroke Spasticity: A Systematic Review and Meta-Analysis. Archives of physical medicine and rehabilitation, 98(12), 2578-2589.e4. https://doi.org/10.1016/ j.apmr.2017.03.023

4. Long, Y. B., \& Wu, X. P. (2012). A meta-analysis of the efficacy of acupuncture in treating dysphagia in patients with a stroke. Acupuncture in medicine : journal of the British Medical Acupuncture Society, 30(4), 291-297. https://doi.org/10.1136/acupmed $-2012-010155$

5. Lee, B. H., Lim, S. C., Lee, K. M., Kim, J. S., \& Jung, T. Y. (2009). A Review on the Seven Acupoints for Stroke. Korean Journal of Acupuncture, 26(3), 103-120. (Korean)

6. Fang, J., Chen, L., Ma, R., Keeler, C. L., Shen, L., Bao, Y., \& Xu, S. (2016). Comprehensive rehabilitation with integrative medicine for subacute stroke: A multicenter randomized controlled trial. Scientific reports, 6, 25850. https://doi.org/10.1038/srep25850

7. Chen, L., Fang, J., Ma, R., Gu, X., Chen, L., Li, J., \& Xu, S. (2016). Additional effects of acupuncture on early comprehensive rehabilitation in patients with mild to moderate acute ischemic stroke: a multicenter randomized controlled trial. BMC complementary and alternative medicine, 16, 226. https://doi.org/ 10.1186/s12906-016-1193-y

8. Sze, F. K., Wong, E., Or, K. K., Lau, J., \& Woo, J. (2002). Does acupuncture improve motor recovery after stroke? A meta-analysis of randomized controlled trials. Stroke, 33(11), 2604-2619. https://doi.org/10.1161/01. 
str.0000035908.74261.c9

9. Moon, S. K., Kim, Y. S., Park, S. U., Jeong U. S., Go, C. N., Jo, G. H., Bae, H. S., Lee, J. D., \& Kim, D. Y. (2004). The Effect of LI4-LI11 Electrical Acupuncture at Unaffected Limb on Cerebral Blood Flow in Ischemic Stroke Patients Using SPECT. Journal of Acupuncture Research, 21(1), 111-118. (Korean)

10. Ryu, J. M., Moon, S. K., Kim, Y. S., Park, S. U., Jung, W. S., Ko, C. N., Cho, K. H., Bae, H. S., \& Kim, D. Y. (2006). Effect of LI4-LI11 Electro-Acupuncture on Regional Cerebral Blood Flow in Healthy Human Evaluated by $99 \mathrm{mTc}-\mathrm{ECD}$ Brain SPECT. Journal of Korean Medicine, 27(2), 36-43. (Korean)

11. Cho, S. Y., Jahng, G. H., Park, S. U., Jung, W. S., Moon, S. K., \& Park, J. M. (2010). fMRI study of effect on brain activity according to stimulation method at LI11, ST36: painful pressure and acupuncture stimulation of same acupoints. Journal of alternative and complementary medicine (New York, N.Y.), 16(4), 489-495. https://doi.org/ 10.1089/acm.2009.0395

12. Hong, J. M., Joo, I. S., Huh, K., \& Sheen, S. S. (2010). Simultaneous vasomotor reactivity testing in the middle cerebral and basilar artery with suboccipital probe fixation device. Journal of neuroimaging : official journal of the American Society of Neuroimaging, 20(1), 83-86. https://doi.org/10.1111/j.1552-6569.2008. 00353.x

13. Panerai R. B. (2009). Transcranial Doppler for evaluation of cerebral autoregulation. Clinical autonomic research : official journal of the Clinical Autonomic Research Society, 19(4), 197-211. https://doi.org/10.1007/s10286 $-009-0011-8$

14. Bisschops, R. H., Klijn, C. J., Kappelle, L. J., van Huffelen, A. C., \& van der Grond, J. (2003). Association between impaired carbon dioxide reactivity and ischemic lesions in arterial border zone territories in patients with unilateral internal carotid artery occlusion. Archives of neurology, 60(2), 229-233. https://doi.org/10.1001/archneur.60.2.229

15. Byeon, H. S., Moon, S. K., Park, S. U., Jung, W. S., Park, J. M., Ko, C. N., Cho, K. H., Kim, Y. S., \& Bae, H. S. (2011). Effects of GV20 acupuncture on cerebral blood flow velocity of middle cerebral artery and anterior cerebral artery territories, and $\mathrm{CO}_{2}$ reactivity during hypocapnia in normal subjects. Journal of alternative and complementary medicine (New York, N.Y.), 17(3), 219-224. https://doi.org/10.1089/acm.2010.0232

16. Im, J. W., Moon, S. K., Jung, W. S., Cho, K. H., Kim, Y. S., Park, T. H., Ko, C. N., Park, J. M., Park, S. U., \& Cho, S. Y. (2014). Effects of acupuncture at $\mathrm{GB} 20$ on $\mathrm{CO}_{2}$ reactivity in the basilar and middle cerebral arteries during hypocapnia in healthy participants. Journal of alternative and complementary medicine (New York, N.Y.), 20(10), 764-770. https://doi.org/10.1089/acm. 2013.0240

17. Hyun, S. H., Im, J. W., Jung, W. S., Cho, K. H., Kim, Y. S., Ko, C. N., Park, J. M., Park, S. U., Cho, S. Y., \& Moon, S. K. (2014). Effect of ST36 Acupuncture on Hyperventilation -Induced $\mathrm{CO}_{2}$ Reactivity of the Basilar and Middle Cerebral Arteries and Heart Rate 
Variability in Normal Subjects. Evidence-based complementary and alternative medicine : eCAM, 2014, 574986. https://doi.org/10.1155/ 2014/574986

18. Moon, S. K., Kwon, S., Cho, S. Y., Park, S. U., Jung, W. S., Park, J. M., Ko, C. N., Cho, K. H., \& Son, J. H. (2019). Effects of GB34 acupuncture on hyperventilation-induced carbon dioxide reactivity and cerebral blood flow velocity in the anterior and middle cerebral arteries of normal subjects. Acupuncture in medicine : journal of the British Medical Acupuncture Society, 37(5), 277-282. https://doi.org/10.1136/acupmed-2017-011474

19. Zhao, L., Chen, J., Liu, C. Z., Li, Y., Cai, D. J., Tang, Y., Yang, J., \& Liang, F. R. (2012). A review of acupoint specificity research in china: status quo and prospects. Evidence -based complementary and alternative medicine : eCAM, 2012, 543943. https://doi.org/10. $1155 / 2012 / 543943$

20. MacPherson, H., Altman, D. G., Hammerschlag, R., Li, Y., Wu, T., White, A., Moher, D., \& STRICTA Revision Group (2010). Revised STandards for Reporting Interventions in Clinical Trials of Acupuncture (STRICTA): extending the CONSORT statement. Acupuncture in medicine : journal of the British Medical Acupuncture Society, 28(2), 83-93. https://doi.org/ 10.1136/aim.2009.001370

21. Markwalder, T. M., Grolimund, P., Seiler, R. W., Roth, F., \& Aaslid, R. (1984). Dependency of blood flow velocity in the middle cerebral artery on end-tidal carbon dioxide partial pressure--a transcranial ultrasound Doppler study. Journal of cerebral blood flow and metabolism : official journal of the International Society of Cerebral Blood Flow and Metabolism, 4(3), 368-372. https://doi.org/ $10.1038 / \mathrm{jcbfm} .1984 .54$

22. Izumi, Y., Tsuda, Y., Ichihara, S., Takahashi, T., \& Matsuo, H. (1996). Effects of defibrination on hemorheology, cerebral blood flow velocity, and $\mathrm{CO} 2$ reactivity during hypocapnia in normal subjects. Stroke, 27(8), 1328-1332. https://doi.org/10.1161/01.str.27.8.1328

23. Ringelstein, E. B., Sievers, C., Ecker, S., Schneider, P. A., \& Otis, S. M. (1988). Noninvasive assessment of $\mathrm{CO} 2$-induced cerebral vasomotor response in normal individuals and patients with internal carotid artery occlusions. Stroke, 19(8), 963-969. https://doi.org/10.1161/01.str.19.8.963

24. Sakashita, Y., Kanai, M., Sugimoto, T., Taki, S., \& Takamori, M. (1997). Changes in cerebral blood flow and vasoreactivity in response to acetazolamide in patients with transient global amnesia. Journal of neurology, neurosurgery, and psychiatry, 63(5), 605-610. https://doi.org/10. 1136/jnnp.63.5.605

25. White, R. P., Deane, C., Vallance, P., \& Markus, H. S. (1998). Nitric oxide synthase inhibition in humans reduces cerebral blood flow but not the hyperemic response to hypercapnia. Stroke, 29(2), 467-472. https://doi.org/10.1161/ 01.str.29.2.467

26. White, R. P., Deane, C., Hindley, C., Bloomfield, P. M., Cunningham, V. J., Vallance, P., Brooks, D. J., \& Markus, H. S. (2000). The effect of the nitric oxide donor glyceryl trinitrate on global and regional cerebral blood flow in man. Journal of the 
neurological sciences, 178(1), 23-28. https://doi.org/ $0.1016 / \mathrm{s} 0022-510 \mathrm{x}(00) 00357-9$

27. Leung, S. B., Zhang, H., Lau, C. W., \& Lin, Z. X. (2016). Attenuation of blood pressure in spontaneously hypertensive rats by acupuncture was associated with reduction oxidative stress and improvement from endothelial dysfunction. Chinese medicine, 11(1), 38. https://doi.org/10. 1186/s13020-016 -0110-0

28. Kim, J. I., Kim, Y. S., \& Kim, C. H. (2001). Difference in NOS between $2 \mathrm{~Hz}$ and $100 \mathrm{~Hz}$ EA in cerebral cortex, brain stem and cerebellum of spontaneously hypertensive rats. Journal of Acupuncture Research, 18(4), 116-124. (Korean)

29. Lee, Y. Y., Shin, J. C., Na, C. S., Ryu, C. R., \& Cho, M. R. (2003). Experimental Study of Acupuncture at Haenggan(LR2) on the Cerebral Hemodynamics in Normal Rats. Korean Journal of Acupuncture, 20(4), 31-40. (Korean)
30. Jeong, H. W., Kim, J. W., Han, Y. S., \& Choi, C. H. (2014). Effects of Acupuncture at the Wijung (BL40) and Chok-Samni (ST 36) on the Cerebral Blood Flow and Blood Pressure. Journal of Physiology \& Pathology in Korean Medicine, 28(6), 601-606. (Korean)

\section{ORCID}

Gyeong-muk Kim https://orcid.org/0000-0003-1371-0979

Woo-Sang Jung https://orcid.org/0000-0001-7355-7684

Seungwon Kwon https://orcid.org/0000-0002-1857-3515

Chul Jin https://orcid.org/0000-0002-4646-3495

Seung-Yeon Cho https://orcid.org/0000-0003-3149-9759

Seong-Uk Park https://orcid.org/0000-0002-4617-2719

Sang-Kwan Moon https://orcid.org/0000-0003-0497-3080

Jung-Mi Park https://orcid.org/0000-0002-0851-4431

Chang-Nam Ko https://orcid.org/0000-0002-4749-7961

Ki-Ho Cho https://orcid.org/0000-0001-5824-9114 